\section{Neonates at risk of medium-chain acyl-CoA dehydrogenase deficiency: a perinatal protocol for use before population neonatal screening test results become available}

To the Editor: Since the 1990s, the use of tandem mass spectrometry in population neonatal blood-spot screening (NBS) programs has improved the prognosis for many patients with treatable inherited metabolic diseases. However, clinical awareness among pediatricians and neonatologists remains as important as ever because severe neonatal clinical symptoms may present before population NBS test results are available. ${ }^{1}$ In their article "Morbidity and Mortality Among Exclusively Breastfed Neonates With Medium-Chain Acyl-CoA Dehydrogenase Deficiency," Ahrens-Nicklas et al. ${ }^{2}$ highlight the importance of feeding regimens for these neonates at risk. The authors retrospectively studied a cohort of patients with medium-chain acyl-CoA dehydrogenase (MCAD) deficiency (MIM 201450) and found that $23.9 \%(11 / 46)$ of the infants were symptomatic before the return of NBS results and four of them died or experienced cardiac arrest. All symptomatic neonates had been exclusively breastfed. This is important because it may not be known to pediatricians and neonatologists that a substantial percentage of newborns with MCAD deficiency are symptomatic during the first days of life. In addition, the authors note that six neonates with an older sibling known to have MCAD deficiency were carefully monitored and five of them received supplemental feeding. None of the six carefully monitored neonates experienced metabolic decompensation.

After the pilot NBS study in our region of the Netherlands in 2003-2005, we developed a perinatal protocol for health-care providers and families to monitor neonates at increased risk of MCAD deficiency (Table 1). Parents are instructed to contact the metabolic team near the end of the first trimester of a new pregnancy. Thereafter, an information letter is shared with the parents and the health-care providers (including general practitioner midwife, obstetrician, and pediatrician, when applicable). The letter describes the perinatal monitoring protocol and provides the contact details for our pediatric metabolic team for consultation, which is available 24 hours a day, 7 days a week. Depending on the clinical situation, parents either call us within 24 hours after birth or provide our contact information to the involved health-care providers. In agreement with the report by Ahrens-Nicklas et al., ${ }^{2}$ our protocol emphasizes the importance of a guaranteed minimal intake in milliliters in the first days of life. However, to date we have not communicated any preference regarding formula milk versus mother's milk obtained after breast pumping.

In the Dutch population NBS program in 2013, only $62 \%$ of the positive neonates were referred to a metabolic physician before day 8 . Therefore, our protocol includes analysis of dried blood-spot acylcarnitines before NBS test results can become available. Usually, the dried blood spots are delivered to our center by family members on the second day of life and the results of the acylcarnitine analysis are reported the same day. Although not formally studied, parents reported to us that the protocol substantially reduces the stress regarding the diagnosis and feeding regimen while they await the results of the national NBS program. Table 2 presents acylcarnitine concentrations in umbilical cord blood and the first days of life of two (c.985A $>\mathrm{G}$ ACADM homozygote) MCAD-deficient neonates from families in which the index patients were previously identified by the NBS program. These results illustrate that cord-blood acylcarnitines may enable rapid biochemical diagnosis of these newborns. In our (still relatively limited) experience, neonates in whom MCAD deficiency was excluded have normal (ratios of) acylcarnitines in umbilical cord blood.

Walter et al. studied the overall feasibility of acylcarnitine and amino acid analysis via tandem mass spectrometry in umbilical cord blood for the identification of inherited metabolic diseases. ${ }^{3}$ The authors mentioned several limitations of umbilical cord blood metabolite screening. First, depending on the nature of the metabolic defect and the role of enteral feeding and fasting, only a few inherited metabolic diseases can be detected. ${ }^{3}$ However, the feasibility of more rapid identification also depends on the nature of the disorder. Walter et al. used cutoff values only for acylcarnitine concentrations, but using their molar ratios as well might increase the detection rate of some disorders. ${ }^{3}$ Despite reports of agerelated variations in acylcarnitine concentrations comparing umbilical cord blood samples with NBS samples, ${ }^{4,5}$ dried cord blood-spot acylcarnitine reference values for concentrations including their molar ratios are not yet available. Second, theoretically umbilical blood acylcarnitines can be influenced by maternal (nutritional and/or disease) status. ${ }^{3}$ This problem can be circumvented by sequential acylcarnitine (ratio) analysis. Therefore, in addition to the regular NBS program, acylcarnitine concentrations in umbilical cord blood may provide an early indication.

The abnormal umbilical cord blood acylcarnitine profiles in MCAD-deficient neonates may indicate the importance of mitochondrial fatty-acid oxidation prenatally and/or during the perinatal transition. This period is characterized by important changes in cardiac energy metabolism (i.e., from predominantly carbohydrate-driven toward mitochondrial fatty 
Table 1 Perinatal protocol for neonates at risk of MCAD deficiency pending population NBS results

\footnotetext{
- Before birth:

- No reason for invasive antenatal procedures (treatable, NBS)

- Instruction letter to the parents, general practitioner, midwife, obstetrician, pediatrician

- After birth:

- Clinical observations: body temperature, feedings (alert, vomiting), hypoglycemia

- Feeding regimen: ensure (formula) milk intake by bottle feeds until growth (day $1.8 \times 10 \mathrm{ml}$, day $2.8 \times 20 \mathrm{ml}$, day $3.8 \times 30 \mathrm{ml}$, etc.)

- Parents contact the metabolic pediatrician within $24 \mathrm{~h}$ after birth

Diagnostics:

- Dried blood-spot acylcarnitines: cord blood, day 0 before first or second meal and day 1 before a meal

- Dutch population NBS program
}

Day 0 is defined as the day of birth.

MCAD, medium-chain acyl-CoA dehydrogenase; NBS, neonatal blood-spot screening.

acid-driven) and by changes from passive fetal toward active neonatal glucose homeostasis and thermoregulation.

To summarize, a substantial percentage of newborns with MCAD are symptomatic during the first days of life. Therefore, a perinatal protocol is important and reduces the risk of early clinical ascertainment. Analysis of umbilical dried bloodspot acylcarnitines (and their molar ratios) can enable rapid diagnosis.

\section{DISCLOSURE}

The authors declare no conflict of interest.

Willemijn J. van Rijt, BSc, ${ }^{1}$, Emmalie A. Jager, BSc, ${ }^{1}$,

Francjan J. van Spronsen, $M D, P h D^{1}$, Tom de Koning, $M D, P h D^{1}$, M. Rebecca Heiner-Fokkema, $P h D^{2}$ and Terry G.J. Derks, $M D, P h D^{1}$
Table 2 Dried blood-spot acylcarnitine profiles of two neonates with MCAD deficiency

\begin{tabular}{|c|c|c|c|c|c|}
\hline & $\begin{array}{c}\mathrm{CO} \\
(\mu \mathrm{mol} / \mathrm{l})\end{array}$ & $\begin{array}{c}\text { C8 } \\
(\mu \mathrm{mol} / \mathrm{l})\end{array}$ & $\begin{array}{c}\text { C10 } \\
(\mu \mathrm{mol} / \mathrm{l})\end{array}$ & $\begin{array}{c}\text { C10:1 } \\
(\mu \mathrm{mol} / \mathrm{l})\end{array}$ & $\begin{array}{c}\mathrm{C} 8 / \mathrm{C} 10 \\
\text { ratio }\end{array}$ \\
\hline \multicolumn{6}{|c|}{ NBS thresholds } \\
\hline $72-168 \mathrm{~h}$ & - & $\geq 0.5$ & _- & _- & $\geq 5.0$ \\
\hline \multicolumn{6}{|l|}{ Case A } \\
\hline Cord blood & 28 & 0.59 & - & - & 10.3 \\
\hline Day 0 & 45 & 1.45 & - & - & 16.1 \\
\hline Day 1 & 50 & 9.7 & 0.55 & 0.34 & 17.8 \\
\hline NBS results & 14.8 & 7.98 & 0.51 & - & 15.6 \\
\hline \multicolumn{6}{|l|}{ Case B } \\
\hline Cord blood & 30 & 0.64 & 0.06 & 0.05 & 12.8 \\
\hline Day 0 & 36 & 4.27 & 0.29 & 0.11 & 14.7 \\
\hline Day 1 & 40 & 9.81 & 0.63 & 0.27 & 15.6 \\
\hline NBS results & - & 1.77 & 0.15 & - & 11.8 \\
\hline
\end{tabular}

Day 0 is defined as the day of birth.

MCAD, medium-chain acyl-CoA dehydrogenase; NBS, neonatal blood-spot screening.

${ }^{1}$ Section of Metabolic Diseases, Beatrix Children's Hospital, University of Groningen, University Medical Center Groningen, Groningen, The Netherlands; ${ }^{2}$ Laboratory of Metabolic Diseases, Department of Laboratory Medicine, University of Groningen, University Medical Center Groningen, Groningen, The Netherlands; Correspondence: Terry G.J. Derks (t.g.j.derks@umcg.nl)

\section{REFERENCES}

1. van Rijt WJ, Koolhaas GD, Bekhof J, et al. Inborn errors of metabolism that cause sudden infant death: a systematic review with implications for population neonatal screening programmes. Neonatology 2016;109:297-302.

2. Ahrens-Nicklas RC, Pyle LC, Ficicioglu C. Morbidity and mortality among exclusively breastfed neonates with medium-chain acyl-CoA dehydrogenase deficiency. Genet Med; e-pub ahead of print 5 May 2016.

3. Walter JH, Patterson A, Till J, Besley GT, Fleming G, Henderson MJ. Bloodspot acylcarnitine and amino acid analysis in cord blood samples: efficacy and reference data from a large cohort study. J Inherit Metab Dis 2009;32:95-101.

4. Meyburg J, Schulze A, Kohlmueller D, Linderkamp O, Mayatepek E. Postnatal changes in neonatal acylcarnitine profile. Pediatr Res 2001;49:125-129.

5. Cavedon CT, Bourdoux P, Mertens K, et al. Age-related variations in acylcarnitine and free carnitine concentrations measured by tandem mass spectrometry. Clin Chem 2005;51:745-752.

Advance online publication 22 September 2016. doi:10.1038/gim.2016.149 